\title{
EDITORIAL \\ Una visión del manejo de residuos sólidos en Iberoamérica
}

El desarrollo de la humanidad ha implicado acceso a formas cada vez más variadas y vinculadas con sus prácticas sociales. En el siglo XXI la humanidad ha visto crecer sus necesidades de insumos con gran rapidez, estos satisfactores que demanda la sociedad del siglo XXI son provistos a través de procesos de industrialización y comercialización, lo que representa una notoria explotación de los recursos naturales y la generación de grandes volúmenes de desechos.

Es así que el manejo de residuos sólidos es un tema de interés mundial, por ello en este volumen se presenta una visión del manejo de residuos sólidos en Iberoamérica, resultado del trabajo en red realizado por un grupo de investigadores de algunos países de esta región. Contiene 12 artículos, que analizan diversos temas del manejo de residuos, se incluye un artículo en el que se presenta un análisis y comparación de la composición de residuos sólidos domiciliarios (RSD) en ciudades de cinco países de Latinoamérica. Entre los hallazgos, los autores de este articulo encontraron variaciones en la generación y composición de los RSD de las ciudades estudiadas, observaron que la composición es más heterogénea, así mismo reportan que los subproductos reciclables están presentes en el flujo de RSD, siendo la fracción orgánica la que continua predominando. También destacan la importancia de contar con datos precisos y actualizados de tasas de generación y composición, ya que estos datos son esenciales para el diseño de programas de gestión que incluyan el reciclaje y la disposición final adecuada.

En el segundo artículo, se hace una revisión de la aplicación de la metodología de Análisis de Ciclo de Vida (ACV) como una herramienta para evaluar el desempeño ambiental de los sistemas de gestión de residuos sólidos. Analizan cinco experiencias de aplicación de la metodología en las que evalúan el comportamiento ambiental de un sistema de recogida selectiva de residuos domiciliarios en España; un sistema de gestión de residuos de aparatos eléctricos y electrónicos y específicamente terminales de telefonía móvil en México; un sistema de gestión de residuos de construcción y demolición en Argentina; la incorporación de recogida selectiva en un sistema de gestión de residuos domiciliarios en Brasil; y finalmente, un sistema de gestión de residuos domiciliarios en Colombia. Señalan la importancia de contar con inventarios en bases de datos comerciales adaptados a diferentes países, fracciones y tratamientos de gestión de residuos, para obtener mejores resultados.

En el tercer artículo se presenta la evaluación de riesgos (ER) como una herramienta aplicada al manejo de residuos sólidos urbanos (RSU) y a la operación de sitios de disposición final (SDF), se establece que esta herramienta puede ayudar a estimar y jerarquizar los riesgos ocasionados por esos sitios. La información que se obtiene de ER proporciona elementos para la elaboración de medidas normativas de prevención de riesgos, así como en la toma de decisiones de control ambiental, a través del suministro de información científica.

En el cuarto artículo, los autores realizan un análisis de los plásticos, una de las corrientes de residuos que está en continuo crecimiento y provoca efectos ambientales por la saturación de sitios de disposición final y afectaciones en los ecosistemas por su manejo inadecuado. Se analizan y comparan la generación de residuos plásticos no 
industriales, las medidas legislativas enfocadas en la minimización de sus impactos y el aumento de su valorización.

También se ha incluido un artículo que analiza el desarrollo de la industria del biodiesel en Colombia y México, a partir de la utilización de residuos. Los autores comparan esta industria en ambos países y reportan que para producir biodiesel, en Colombia, se utiliza una tecnología que procesa palma la cual genera residuos que son aprovechados como fuente de energía en otros procesos, reportan que el desarrollo de la industria del biodiesel en este país se debe a la integración de los productores de palma quienes proveen las materias primas. El trabajo articulado de todos los actores, provee y asegura la plataforma y condiciones de mercado del biodiesel. El reto para Colombia es continuar con esta dinámica y explorar otras materias primas tales para producir biodiesel. En el caso de México, las tecnologías de las plantas fueron diseñadas para procesar residuos y semillas oleaginosas.

Otro tema que se aborda en este número es el de rellenos sanitarios, en tres artículos, se presenta una revisión del estado actual del conocimiento sobre biorrellenos. Los autores reúnen las directrices generales de diseño y operación recomendables para nuevas instalaciones de biorrellenos anaeróbicos y semiaeróbicos, incluyendo los sistemas de recirculación de lixiviados y extracción del gas generado, los cambios principales respecto a la operación de un relleno tradicional y las necesidades de seguimiento. Analizan una experiencia a escala real, un modelo de biorrelleno semiaeróbico aplicable a países de desarrollo intermedio y dos casos representativos del avance en este campo en Iberoamérica.

Otro tema que se aborda en relleno sanitario relleno sanitarios es el de los rellenos sanitarios constituyen una fuente importante de biogás resultado del proceso de descomposición biológica de residuos sólidos de origen orgánico.

Se aborda el tema de la generación de biogás en un relleno sanitario para ello se comparan los modelos de biogás aplicándolos a datos empíricos obtenidos en la medición de la generación de biogás entre 2009 y 2011 de un relleno sanitario situado en el municipio de Zapopan, en el estado de Jalisco (México) analizando el grado de coincidencia entre los modelos y el relleno. Los autores identifican cuales son los modelos que sobreestiman, y cuales subestiman la generación de biogás, asi como los modelos que se alejan demasiado de las mediciones reales, por lo que proponen que debería descartar su uso en el relleno bajo estudio. Los resultados de la comparación muestran que el Modelo Mexicano, el EPER Francés y el SWANA Orden Cero son los que más se aproximan.

En otro artículo, sobre el tema de rellenos sanitarios, los autores hacen una revisión de las experiencias en Iberoamérica sobre el proceso de cierre, sellado y reinserción de antiguos vertederos, recopilando informaciones técnicas, propone la metodología básica a seguir en un proyecto de este tipo y presenta varios ejemplos de clausura y reinserción de vertederos en esta parte del mundo.

El último tema que se aborda, es tratamiento, se incluyen cuatro trabajos, el objetivo de uno de los artículos que abordan este tema es presentar una revisión de las investigaciones realizadas principalmente en Iberoamérica, en relación a la utilización de diversos subproductos biológicos en el cultivo y producción de los hongos comestibles (Pleurotus spp.). Anlizan una amplia variación en eficiencias biológicas (EB) (15\% al 235\%) obtenidas en los diferentes estudios y, de manera sintética, los criterios de elegibilidad de los materiales.

Luego se incluye un artículo, que presenta un estudio de caso de uso de procesos aerobios para tratar residuos orgánicos con alto contenido de humedad y bajo contenido de lignina y celulosa. Los autores usaron una mezcla de viruta de madera y lirio acuático seco (Eichhornia crassipes) como agente estructurante en diferentes porcentajes $(5,10,15$ y $20 \%)$. Los resultados que presentan muestran que el elevado contenido de humedad de la mezcla puede ser regulado con materiales estructurantes secos.

Se incluye otro estudio de caso para el tratamiento de residuos de jardín de un campus universitario, para ello los autores aplicaron un proceso de secado biológico a estos residuos. Analizaron la influencia que tiene la adición de un agente texturizante en el proceso. Los parámetros que influyeron en el proceso fueron: aireación, temperatura y humedad inicial del biorresiduo. Los autores señalan que el aumento del caudal de aire proporcionado al reactor no es linealmente proporcional a la pérdida de peso del sustrato. 
En el último artículo presentan los resultados de un análisis de indicadores inorgánicos y actividad enzimática de tres mezclas de vermicomposta hechas con estiércol bovino (EB), residuos domésticos (RD), lodo residual (LR) y suelo (Cambisol), para conocer su madurez. Entre las conclusiones que reportan señalan que la presencia del lodo residual y la misma proporción de estiércol bovino y residuos domésticos en la elaboración de la vermicomposta potencializa la estabilidad de la materia orgánica y llega a una madurez en el producto elaborado.

Los trabajos que se presentan en este volumen especial, aportan información valiosa sobre el tema de los residuos sólidos en Iberoamérica, como ya se mencionó abarcan varias áreas, además es importante destacar que la interrelación académico científica que se ha establecido entre miembros de redes que a su vez pertenecen a diversas universidades enriquece la visión y el contenido de los artículos que se presentan.

Dr. Antonio Gallardo Izquierdo Universidad Jaime 1, España Líder RED REDIGAR/CYTED Editor Invitado

Dra. Samantha E. Cruz Sotelo Facultad de Ingeniería Universidad Autónoma de Baja California Miembro fundador de SOMERS Editora Invitada

Dra. Sara Ojeda Benítez Instituto de Ingeniería, UABC Editora Asociada 